\title{
Nikolaus Senn (1844-1908), ein schweizerischer Pionier der Chirurgie in den USA
}

\author{
Von Heinrich Buess
}

Das Urbar des Kirchspiels Buchs im St. Galler Rheintal weist im Jahre 1484 zum erstenmal den Namen Senn auf. Im öffentlichen Leben trat das Geschlecht jedoch erst im 19. Jahrhundert hervor. Unter seinen Vertretern befinden sich mehrere Lehrer, die sich teilweise dank ihrer vielseitigen Begabung zu bedeutenden Stellungen emporarbeiteten. Einer von ihnen ging schon in jungen Jahren nach Amerika und wurde Senator des Staates Kansas. Der Vater unseres Arztes scheint Landwirt gewesen zu sein.

\section{I}

Nikolaus kam am 31.Oktober 1844 in Buchs zur Welt. Im Jahre 1853 wanderte die Familie nach Nordamerika aus. Es war dies die Zeit, als die teilweise drückende Lage der schweizerischen Landwirtschaft manchen Bauern dazu verlockte, in der Neuen Welt mit ihren vielen noch nicht erschlossenen Bodenschätzen sein Glück zu versuchen. Die Familie Senn fand in Ashford (Wisconsin) eine neue Heimstätte. In diesem Staat entstand eine eigentliche Schweizer Kolonie (New Glarus usw.). Durch rastlose Arbeit in einer Gegend, die damals noch an der Grenze der Zivilisation lag, gelang es dem unternehmenden St. Galler, dort bald festen Fuß zu fassen und sich über die ersten wirtschaftlichen Schwierigkeiten hinweg zu einem gewissen Wohlstand aufzuschwingen.

Dem Sohn wurde jedenfalls, gemessen an den primitiven Verhältnissen, eine sorgfältige Erziehung zuteil. Er besuchte die Sekundarschule eines benachbarten Städtchens. Seine hervorragenden Geistesgaben, sein ausgezeichnetes Gedächtnis und die Leidenschaft des aufgeweckten Jünglings für naturwissenschaftliche Beobachtungen ließen schon früh Außergewöhnliches erwarten. Zunächst wurde Senn Volksschullehrer in Fond du Lac (Wisconsin). In dieser bescheidenen Stellung trieb er botanische Studien, zu denen ihm die reichhaltige Flora der teilweise noch nicht kultivierten ländlichen Umgebung mannigfache Gelegenheit bot. Sein Genie brach sich jedoch Bahn, denn Senn war für Höheres bestimmt. Bald regte sich das Interesse für den ärztlichen Beruf, und in der Freizeit erteilte ihm der Arzt von Fond du Lac, Dr. E. Munk, privaten Unterricht.

Nach zwei Jahren hielt es den 21 jährigen nicht mehr länger an seinem bescheidenen Posten. Er begann das eigentliche Medizinstudium an der Medizinschule in Chicago. 1868 wurde er mit höchster Auszeichnung zum Arzt diplomiert, 
nachdem er eine Arbeit über die Wirkung der Digitalis purpurea (Fingerhut) eingereicht hatte. Kurz darauf verheiratete er sich und gründete im heimatlichen Ashford eine Praxis. Dorthin war er wohl auf den Wunsch seiner Eltern gegangen, die ihren geliebten und hoffnungsvollen Sohn in der Nähe haben wollten. Der glänzend vorgebildete Arzt - war er doch während 18 Monaten Assistent von Professor William Quine am Cook County-Hospital in Chicago gewesen - brauchte nicht lange auf Patienten zu warten. Doch bald wurde ihm das Wirkungsfeld zu klein, und schon 1873 übersiedelte er nach Milwaukee, der hauptsächlich von Deutschen bewohnten, schon damals bedeutendsten Stadt von Wisconsin. Hier konnte sich sein Talent voll entfalten. Zur Erweiterung seiner Kenntnisse begab er sich 1877/78 nach Europa, das damals den Siegeszug der antiseptischen Chirurgie erlebte, und er erwarb sich in München die Doktorwürde. Diese Reisen brachten es mit sich, daß Senn zum Vermittler zwischen der europäischen und der amerikanischen Forschung wurde. Insbesondere in der deutschsprachigen Literatur, die er dank seiner Herkunft mühelos beherrschte, war er zu Hause wie kaum ein zweiter Chirurg in den USA.

In Chicago, der aufblühenden Industrie- und Handelsstadt, wurde er bald als Spitalchirurg zugezogen. Dort fand er die nötige wissenschaftliche Anregung im Kreise der Kollegen. Bald war es indessen Senn, der die geistige Führung der dortigen Ärzteschaft übernahm. Die Beschäftigung mit der modernen europäischen Forschung hatte ihn eines vor allem erkennen lassen: die überragende Bedeutung des Tierexperiments. Nach seiner Rückkehr ging er denn mit vermehrter Energie daran, den Tierversuch auch der Chirurgie dienstbar zu machen. Seine aufreibende Tätigkeit hinderte Senn nicht daran, den Wert der neu eingeführten chirurgischen Operationen im Experiment zu prüfen und Studien über Komplikationen nach chirurgischen Eingriffen vorzunehmen. Nächtelang konnte der Unverwüstliche, zusammen mit M. E. Connel, über seinen Versuchen sitzen, um in immer wieder neuen Anordnungen dem Ziel näher zu kommen. Und stets war ihm daran gelegen, seine Erfahrungen auch den Kollegen nutzbar zu machen. Einer der älteren Ärzte erzählte von ihm: «Der junge Senn kam nie ohne ein großes Manuskript in die medizinische Gesellschaft. Dieses enthielt jedoch nicht weitschweifige theoretische Erörterungen, sondern steckte voll wertvoller Berichte über experimentelle Beobachtungen aus seinem Laboratorium und Tierstall. Senn demonstrierte seine Entdeckungen mit einem solchen Enthusiasmus und einer derartigen Überzeugungskraft, daß man ihm nicht widerstehen konnte, und wir alle gingen mit neuen Anregungen für unser Denken und Forschen heim aus der Sitzung.»

Dieses ausgezeichnete Lehrtalent trug Senn im Jahre 1884 die Berufung als 
Professor der allgemeinen Chirurgie an die Medizinschule von Chicago ein. Auch dort war er bald bei den Studenten besonders beliebt. Und selbst praktische Ärzte wurden durch seine klare, geradezu klassische Sprache und die überzeugenden Demonstrationen aufschlußreicher Fälle zum häufigen Besuch der Klinik veranlaßt. Ja sogar Senns verehrter Freund Christian Fenger (1840-1902), der Senior der amerikanischen Chirurgen, ließ es sich später nicht nehmen, einzelnen Vorlesungen beizuwohnen. Das erstaunlichste an der Leistung Senns scheint jedoch die Tatsache zu sein, daß er anfangs ein-, später zweimal wöchentlich etwa $150 \mathrm{~km}$ zurücklegte, um in Chicago diesen Pflichten als Professor nachzukommen. Auf der langen Eisenbahnfahrt entlang dem Michigansee entstanden die mit soviel Beifall aufgenommenen prachtvollen Vorträge. Erst im Jahre 1893, nachdem er schon früher zum Professor am berühmten Medical College ernannt worden war, nahm Senn seinen Wohnsitz in Chicago selbst.

Der Wechsel brachte noch größere Verpflichtungen mit sich: Senn wurde Chefarzt des großen St. Josephsspitals und bald auch konsultierender Chirurg an anderen Kliniken. Schon 1891 war ihm die Nachfolge von Ch.T.Parks auf dem Lehrstuhl der praktischen Chirurgie übertragen worden. Damit hatte Senn innert kürzester Zeit die höchste Stufe seiner raschen Karriere erklommen. Jetzt besa $\beta$ er die Möglichkeit, seine experimentell erprobten Methoden in die Praxis umzusetzen und damit Tausenden von Menschen das Leben zu retten. Von den verschiedenen Biographen wird der einstige Rheintaler als der bedeutendste amerikanische Chirurg seiner Zeit bezeichnet. Senn wurde für eine ganze Generation von Ärzten zum leuchtenden Vorbild als Mensch, der sich neben seinem Beruf kaum ein Vergnügen gönnte, als Arzt, der sich im Dienste seiner Kranken aufzehrte, als Lehrer, dessen Worte weithin gehört wurden, und als Forscher, der seine Beobachtungen und Erfahrungen in zahlreichen Veröffentlichungen allgemein bekannt machte.

Senns besonderes Interesse galt schon in jungen Jahren dem Militärwesen und besonders der Militärchirurgie. Nachdem er schon in Wisconsin Generalarzt geworden war (1888), wurde Senn vom Gouverneur von Illinois ebenfalls zum höchsten Militärchirurgen ernannt. In dieser Stellung setzte er eine strenge physische und geistige Prüfung der Rekruten durch, führte eine geordnete Ausbildung der Offiziersanwärter der Sanitätstruppe ein - Senn war später auch Professor der Militärchirurgie - und stellte die Bedingungen auf für die Erlangung des Patents als Sanitätsoffizier. Indessen erschöpfte sich Senns Tätigkeit nicht in diesen Fragen der Friedensorganisation, sondern er nahm als aktiver Chirurg zuerst am griechisch-türkischen (1897) und ein Jahr später am spanischamerikanischen Krieg um Kuba teil. Als Generalarzt des 6. Armeekorps und als 
Leiter des Lazarettwesens erwarb er sich im Sommerfeldzug besondere Verdienste, so daß der Name von Oberstleutnant Senn in einem Armeebefehl erwähnt wurde. In einem größeren, zweimal aufgelegten Werk sind seine Erfahrungen als Armeechirurg niedergelegt.

Wie groß das Ansehen Senns auf internationalem Gebiet war, beweist seine Teilnahme als offizieller Delegierter an verschiedenen internationalen medizinischen Kongressen, so in Berlin (1890), Moskau (1897) u.a. Auch war er Ehrenmitglied der Londoner Chirurgen-Gesellschaft, zahlreicher skandinavischer Vereinigungen und einer kaum übersehbaren Reihe von amerikanischen Fachgesellschaften. Im damaligen Herausgeber-Kollegium der großen Zeitschrift «Surgery, Gynecology and Obstetrics» steht Senns Name obenan. Denn abgesehen davon, daß er in der gesamten medizinischen Literatur wie kaum ein zweiter zu Hause war - Murphy vergleicht sein Wissen mit einer medizinischen Buchhandlung -, besaß Senn eine der größten Bibliotheken Amerikas. Durch Kauf hatte er die Bücherei des Göttinger Chirurgen Wilhelm Baum (1799-1883) und des Physiologen Emil Du Bois-Reymond (1818-1896) in Berlin erworben. Er besaß damit einen Grundstock von größtem Wert. Dies äußerte sich auch darin, daß Senn zahlreiche kleinere Arbeiten zur lokalen und allgemeinen Geschichte der Chirurgie verfaßte. Von verschiedenen Reisen datieren seine für die neuere Medizingeschichte aufschlußreichen Berichte über die Zustände des Medizinalwesens in den wichtigsten Städten Europas um die Jahrhundertwende.

Am 11. November 1905 versammelten sich Senns Freunde und Schüler, um ihrem verehrten «Master Surgeon», wie er allgemein hieß, eine goldene Medaille und einen silbernen Pokal zu überreichen. Ob bei diesem feierlichen Anlaß auch der Landsmann des Geehrten, der Schaffhauser Chirurg Martin Stamm (18471918), zugegen war, der als Professor an der Columbia-Universität (Ohio) wirkte, wird nicht gesagt. Die Denkmünze trug die Inschrift: «Nicholas Senn, dem Meister-Chirurgen, von seinen Genossen.» Die Huldigung konnte mit der eines Fürsten verglichen werden, wie Blech sagt. In höchster Rührung und Bescheidenheit nahm Senn die Lobeserhebungen entgegen mit den Worten: «Wenn ich nur etwas für die Wissenschaft tun konnte.» Er war damals schon ein gebrochener Mann. Ein schweres Herzleiden (Myokarditis) zehrte an seinen Kräften. Eine Reise nach Südamerika verschlimmerte die Krankheit. Als sich eine Nierenentzündung dazugesellte, waren die Tage des großen Arztes gezählt, der für unser Land in der neuen Heimat so hohe Ehre eingelegt hat. Er starb am 2. Januar 1908 in Chicago. Welche schöneren Worte könnte man sich denken als die, welche ihm sein berühmter Kollege John B. Murphy (1857-1916) nachrief: «Die Welt ist deshalb besser geworden, weil in ihr ein Senn gelebt hat.» 
Um die Mitte des 19. Jahrhunderts hatte die Chirurgie in den Vereinigten Staaten gewaltige Fortschritte gemacht. Es sei nur an die Entdeckung der Äthernarkose durch Charles T. Jackson (1805-1880) in den vierziger Jahren erinnert, die von Amerika aus die Welt eroberte und heute zum bevorzugten Mittel der Allgemeinbetäubung geworden ist. Der vom Zahnarzt William Morton (1819-1868) applizierte Äther wurde am 16. Oktober 1846 vom Chirurgen John Collins Warren (1778-1856) zum erstenmal bei einer größeren Operation (Entfernung einer Geschwulst am Hals) verwendet. Innert weniger Wochen wurde das Wundermittel den Chirurgen Englands und Frankreichs bekannt. Aber auch auf dem Gebiet der Operationstechnik durften die Amerikaner zahlreiche Ersterfolge für sich buchen. So führte der Landarzt Ephraim McDowell (1771-1830) im Jahre 1809 als erster mit glücklichem Erfolg eine Ovariotomie (Eierstockoperation) aus, eine für die damalige Zeit außerordentlich kühne Tat. Neben der Gynäkologie erfuhr besonders die Chirurgie der Gliedmaßen und der Blutgefäße reiche Förderung durch die Amerikaner. Auch in der Lehre von den Bauchoperationen sind die Namen mehrerer Chirurgen der westlichen Hemisphäre bekannt geworden, so z. B. Charles MacBurney (1845-1913) in der Indikationsstellung für die Blinddarmoperation und der oben zitierte John B.Murphy bei den Darmausschaltungen.

Nikolaus Senns größte Verdienste liegen, was die chirurgische Forschung betrifft, ebenfalls auf dem Gebiet der Bauchchirurgie. Zunächst darf er wohl als einer der Begründer der Chirurgie der Bauchspeicheldrüse (Pankreas) angesprochen werden. Als Grundlage seines Handelns dienten ihm die Experimente am Tier, die ihm die Frage beantworteten, wieviel man von der Drüse ohne Schaden entfernen dürfe. Es zeigte sich, daß die totale Entfernung technisch kaum durchführbar war, abgesehen von der um jene Zeit von Minkowski und Mering entdeckten schweren hormonalen Störung bei dieser Operation. Die experimentellen Beobachtungen erlaubten es dann Senn, für die Eingriffe bei Erkrankungen der Bauchspeicheldrüse des Menschen, z. B. bei der Bildung von Zysten (Wassergeschwülsten), genaue Richtlinien aufzustellen (1885-1886). Murphy nennt seinen verehrten Kollegen den «Vater der Pankreas-Chirurgie».

Senns Tierversuche kamen aber vor allem den um jene Zeit besonders in Wien (Schule von Billroth) entwickelten Magendarmoperationen zugute. Besonders bei Krebs des Magens, wo ein Teil dieses Organs entfernt werden muß, erweist sich die Schaffung eines neuen Magenausgangs als notwendig. Zu diesem Zwecke müssen bestimmte Teile des oberen Dünndarmes an den Magenmund genäht 
werden. Es ist nun zur Herstellung einer möglichst naturgemäßen Passage des. Speisebreies außerordentlich wichtig, welcher Teil des Dünndarmes mit dem Magen in Verbindung gebracht wird. In jahrelangen Versuchen ging Senn diesen Fragen nach und gab abschließend die eingehende Begründung für die einzelnen Verfahren der Enteroanastomosen, wie diese Operationen genannt werden. Seine Erkenntnisse, auch in der Lehre von den Darmausschaltungen, brachte er nicht nur in seiner Praxis zur Anwendung, sondern machte sie in umfangreichen Monographien auch der ärztlichen Mitwelt nutzbar. Besondere Erwähnung verdient ein Verfahren Senns, das sich indessen nicht durchzusetzen vermochte: die Verwendung eines durchlöcherten, entkalkten Knochenplättchens in der Herstellung der Darmanastomosen. Obgleich sich diese der Vereinfachung dienende Methode - an ihre Stelle trat 1892 der Murphysche Knopf - nicht zu behaupten vermochte, so sind doch die von Senn aufgestellten Grundsätze als bleibendes Vermächtnis in die Bauchchirurgie eingegangen. Darin liegt die geschichtliche Bedeutung des großen Werkes (1889) von Senn über dieses wichtige Teilgebiet der Chirurgie. In einem eindrücklichen Exposé berichtete Senn am internationalen Ärztekongreß in Washington (1888) über seine Erfahrungen als Experimentator und als Operateur. Es sei hier noch die von Senn eingeführte Netzplastik zur Sicherung der Darmnaht erwähnt, die später wieder zu Ehren gekommen ist.

Den lebhaften Interessen Senns für die Militärchirurgie entsprachen die auch hier wenigstens im englischen Sprachgebiet führend gewordenen Werke, in denen die erste Hilfe auf dem Schlachtfeld und die Behandlung der Schußwunden besonders eingehend dargelegt sind. Senn setzte sich auch für die operative Behandlung der Magen- und Darmschüsse mit aller Energie ein. Der Nachweis der Perforationsstellen mit Hilfe von Wasserstoffgas fand allerdings keine allgemeine Anerkennung. Wertvoll für den amerikanischen Sanitätsdienst waren vor allem die von Senn erörterten Fragen organisatorischer Natur, verdankte man doch dem kritischen Urteil des begeisterten Generalarztes manche fruchtbringende Anregung.

Unter den übrigen Problemen, denen Senns besondere Aufmerksamkeit galt er war ein wahrhaft universaler Chirurg und Arzt - seien noch die Luftembolie und die Pseudoleukämie, eine bösartige Blutkrankheit, genannt. (Weiteres s. Bibliographie.) Die Versuche über die Verschleppung von Luftblasen im Gefäßsystem, die Senn während längerer Zeit besonders am Pferd durchführte, gehören zu den frühesten Tierversuchen des Landarztes (1885) und brachten in das dunkle Gebiet manche Klärung. Bei der so häufigen Leukämie, einer bösartigen Störung des blutbildenden Apparates, darf Senn für sich die Ehre in Anspruch nehmen (nach Walter von Brunn, 1928), als erster die Röntgenbestrahlung in die 
Therapie dieser Krankheit eingeführt zu haben. Die darüber im Jahre 1903 erfolgte Veröffentlichung zeigt, daß Senn auf dem ganzen Gebiet der Medizin nicht nur belesen war, sondern daß er aus den Fortschritten der Wissenschaft auch die richtigen Schlußfolgerungen zu ziehen vermochte.

In den Kreis seiner ausgedehnten Versuche schloß Senn auch die bakteriologischen Studien ein. Über seine Ergebnisse berichten nicht nur zahlreiche seiner etwa 300 Abhandlungen (besondere Erwähnung verdient darunter die große Monographie über die Knochentuberkulose), sondern auch das amerikanische Standardwerk «Surgical Bacteriology» (1889), das ins Französische übersetzt wurde. Auf deutschem Boden setzte sich besonders Richard von Volkmann (1830-1889) für die Listerschen Ideen ein. Senns Bedeutung auf diesem Gebiet mag aus dem Vergleich Murphys zwischen der deutschen und der amerikanischen Chirurgie erschlossen werden. "Groß, Fenger und Senn bedeuteten für die klinische, experimentelle und bakteriologische Chirurgie in Amerika das, was Langenbeck, Billroth und Volkmann für die deutsche Chirurgie leisteten.»

\section{Bibliographie}

S. Index Catalogue of the Library of the Surgeon-General's Office, United States Army, 1. Serie, vol. XII, Washington 1891 (S. 876), und 2. Serie, vol. XV, Washington 1910 (S. 415-418).

Daraus sind nur die wichtigsten selbständigen Schriften hier aufgeführt.

1. Fractures of the neck of the femur, with special reference to bony union after intracapsular fracture, Philadelphia 1883 (113 S.).

2. Experimental researches on cicatrization in bloodvessels after ligature, Philadelphia 1885 (117 S.).

3. An experimental and clinical study of air-embolism, Philadelphia 1885 (121 S.).

4. The surgical treatment of cysts of the pancreas, Chicago 1885 (158 S.).

5. The surgery of the pancreas, as based upon experiments and clinical researches, Philadelphia 1886 (129 S.). (Deutsche Übersetzung 1888.)

6. Four months among the surgeons of Europe, Chicago 1887 (157 S.). (Briefe an Dr. Chr. Fenger.)

7. An experimental contribution to intestinal surgery with special reference to the treatment of intestinal obstruction, St. Louis 1888 (84 S., 1 Taf.).

8. Experimental Surgery, Chicago 1889 (522 S.).

9. Intestinal Surgery, Chicago 1889 (269 S.).

10. Surgical bacteriology, Philadelphia 1889 (270 S., 12 Taf.). (Französische Übersetzung 1890.)

11. Principles of Surgery, Philadelphia und London 1890 (611 S.).

12. Tuberculosis of bones and joints, Philadelphia und London 1892 (504 S., 7 Taf.).

13. Enterorrhaphy; its history, technique and present status, Chicago 1893. 
14. The pathology and surgical treatment of tumors, Philadelphia 1895 (709 S., 13 Taf.).

15. War correspondence (Hispano-American War), Chicago 1899 (278 S., mehrere Taf. und Pläne); 2. Aufl. 1900. (Spanische Übersetzung 1902.)

16. Practical surgery for the general practitioner, Philadelphia und London 1901. (1133 S.).

17. The therapeutic value of the Röntgen ray in the treatment of pseudoleucaemia, New York 1903 (12 S.).

\section{Biographie}

1. John B.Murphy, Surgery, Gynecology and Obstetrics 6 (1908), S. $115 \mathrm{ff}$. (mit farbigem Porträt).

2. Gustavus M.Blech (Chicago), Deutsche Zeitschrift für Chirurgie 92 (1908), S. 576-578.

3. Journal of the American Medical Association, vol. 50 (1908), S. 144-145 (Porträt).

4. Carl Garrè, Deutsche medizinische Wochenschrift 34 $4^{I}$ (1908), S. $470 \mathrm{f}$. (Porträt und sehr gute, knappgefaßte Würdigung des Landsmannes).

5. A.Allemann, Münchener medizinische Wochenschrift $55^{I}$ (1908), S. 629 f. (mit Porträt).

(Im Correspondenz-Blatt für Schweizer Aerzte ist merkwürdigerweise kein Nekrolog erschienen. Weitere Würdigungen s. Index Catalogue.)

\section{Summary}

Among the Swiss physicians in the U.S.A. Nikolaus Senn (1844-1908) whose family came from the upper valley of the Rhine (canton of St.Gallen) is rather unknown. His parents emigrated 1853 to the State of Wisconsin where the young doctor first practised medicine (since 1873) in Milwaukee. After a journey to Germany (1877-1878) Senn was called to Chicago where he was appointed professor of general surgery (1884) and consulting surgeon at several clinics. As a Surgeon general in Wisconsin (1888) he caused many improvements in the recruitment of medical officers in the army as well as in the State of Illinois (professor of military surgery etc.). He served as a commander of military hospitals in two wars. Senn's most important contributions to modern surgery are the following ones: utilization of experimental research in abdominal operations (especially: pancreas, enteroanastomosis), treatment of gunshot wounds, air embolism, discussion of bacteriological problems in surgery (1889), surgical treatment of tumors (1895), enterorrhaphy (1893).

Prof.Dr.med. Heinrich Buess

Medizinhistorische Bibliothek

Schönbeinstraße 40

4056 Basel 